\title{
Online assessment of patients' views on hospital performances using Rasch model's KIDMAP diagram Tsair-Wei Chien ${ }^{1,2}$, Wen-Chung Wang ${ }^{\dagger}$, Hsien-Yi Wang ${ }^{4}$ and Hung- Jung $\operatorname{Lin} * 5,6$
}

Address: ${ }^{1}$ Department of management, Chi-Mei Medical Center, Taiwan, Republic of China, ${ }^{2}$ Department of Hospital and Health Care Administration, Chia-Nan University of Pharmacy and Science, Tainan, Taiwan, Republic of China, ${ }^{3}$ Assessment Research Center, Hong Kong Institute of Education, Tai Po, Hong Kong, ${ }^{4}$ Department of Nephrology, Chi Mei Medical Center, Taiwan, Republic of China, ${ }^{5}$ Department of Emergency Medicine, Chi-Mei Medical Center, Taiwan, Republic of China and ${ }^{6}$ Department of Biotechnology, Southern Taiwan University of Technology, Taiwan, Republic of China

Email: Tsair-Wei Chien - healthup@healthup.org.tw; Wen-Chung Wang - wcwang@ied.edu.hk; Hsien-Yi Wang - why8@ms61.hinet.net; HungJung Lin* - smile@mail.chimei.org.tw

* Corresponding author †Equal contributors

Published: 31 July 2009

BMC Health Services Research 2009, 9:135 doi:10.1 186/1472-6963-9-135

Received: 13 February 2009

Accepted: 31 July 2009

This article is available from: http://www.biomedcentral.com//472-6963/9//35

(c) 2009 Chien et al; licensee BioMed Central Ltd.

This is an Open Access article distributed under the terms of the Creative Commons Attribution License (http://creativecommons.org/licenses/by/2.0), which permits unrestricted use, distribution, and reproduction in any medium, provided the original work is properly cited.

\begin{abstract}
Background: To overcome the drawback of individual item-by-item box plots of disclosure for patient views on healthcare service quality, we propose to inspect interrelationships among items that measure a common entity. A visual diagram on the Internet is developed to provide thorough information for hospitals.
\end{abstract}

Methods: We used the Rasch rating scale model to analyze the 2003 English inpatient questionnaire data regarding patient satisfactory perception, which were collected from 169 hospitals, examined model-data fit, and developed a KIDMAP diagram on the Internet depicting the satisfaction level of each hospital and investigating aberrant responses with Z-scores and MNSQ statistics for individual hospitals. Differential item functioning (DIF) analysis was conducted to verify construct equivalence across types of hospitals.

Results: 18 of the 45 items fit to the model's expectations, indicating they jointly defined a common construct and an equal-interval logit scale was achieved. The most difficult aspect for hospitals to earn inpatients' satisfaction were item 29 (staff told you about any medication side effects to watch when going home). No DIF in the I8-item questionnaire was found between types of hospitals, indicating the questionnaire measured the same construct across hospitals. Different types of hospitals obtained different levels of satisfaction. The KIDMAP on the Internet provided more interpretable and visualized message than traditional item-by-item box plots of disclosure.

Conclusion: After removing misfit items, we find that the 18-item questionnaire measures the same construct across types of hospitals. The KIDMAP on the Internet provides an exemplary comparison in quality of healthcare. Rasch analysis allows intra- and inter-hospital performances to be compared easily and reliably with each other on the Internet. 


\section{Background}

Many studies have discussed patients' perception about their hospitals and the benefit of listening to other patients' experience when choosing a hospital [1-8]. There has been a rapid increase in websites that allow patients to rate their hospitals $[9,10]$. In recent years almost all healthcare providers have been explicitly required to conduct surveys of their patients' healthcare experience. Data from such surveys have been published in journals or on websites (Leapfrog Group [11] \& Patient Opinion [12]) but they rarely comply with the Web 2.0 requirement to improve communication between people via social-networking technologies [13]. Those surveys often use individual item-by-item box plots to disclose patient views on hospital service quality. They are thus unable to provide hospital staff with aberrant responses for prudence and further improvement in performance of patient-centered satisfaction, nor to help patients choose hospitals according to an overall performance level.

\section{Critiques of traditional Likert-type patient satisfaction surveys}

Web 2.0 has changed the relationship between patients and hospitals $[4,14]$. Critiques of traditional patient satisfaction surveys $[7,15]$ have led to a new emphasis on measuring patients' experiences rather than their satisfaction levels only [16]. The England Picker Institute Europe (or EPIE for short [17]) has created such questionnaires (shown in Table 1) and ask patients to report in detail their experience with a particular provider at a specific point of time by answering questions about whether or not certain processes or events occurred during the course of a specific episode of care [18], rather than just ask patients to rate their care on a Likert-type scale [19].

\section{Inappropriate individual item-by-item box plots of disclosure}

Items in Picker's questionnaire are often analyzed and presented individually, one item at a time (e.g., item-byitem box plots of disclosure as shown in Figure 1). In so doing, global interrelationships between items are invisible [20]. Besides, measurement error in a single item is often very substantial but it is ignored in such an item-byitem analysis. We are therefore concerned with the interrelationship between items when they are, in effect, measuring a single construct. Advanced analysis is required.

Item response theory (IRT) or Rasch measurement [21] provides such an advanced analysis to take into account the interrelationship among items. A newly designed diagram can be provided to accurately report patients' experiences for each hospital.

\section{Objective measurement requirements}

There are three major problems in conventional analyses of Picker's questionnaires: (a) interval scores: Raw scores of
Picker's questionnaires are ordinal but have been mistakenly treated as interval and analyzed with linear factor analysis; (b) graphic diagrams with correlated items: more efforts should be made to provide meaningful and simple diagrams (e.g., Google Image Labeler labels digital photographs according to their content) by making the task a simple game in which contestants (i.e., hospitals in this study) must both collaborate and compete with each other; and (c) aggregate scores: the benchmark reports that present item-by-item results for patients to compare with national results not only fail to help patients understand the overall performance of each hospital with an aggregate score, but also lack an objective metric to quantify the difference between hospitals. Details are described as follows:

\section{(I) Interval and additive scores for comparison}

How to measure individual differences on an interval scale [22-24] is an important issue in health care systems. The United Kingdom [25] has introduced a pay-for-performance contract for family practitioners according to performance with respect to 146 quality indicators covering clinical care for 10 chronic diseases, organization of care, and patient experience. In this contract, interval scores should be ensured beforehand such that they can be meaningfully compared with each practitioner. Stout [26] quoted Lord and Novick [27] as saying, "A major problem of mental test theory is to determine a good interval scaling to impose when the supporting theory implies only ordinal properties".

\section{(2) KIDMAP improvement of communication on the Internet}

KIDMAP (also called Diagnostic Map), derived from Rasch measurement, provides an output for each individual and summarizes expected and unexpected response patterns. The first large-scale implementation of KIDMAP took place in the Los Angeles Independent County School District in the early 1980s. Hundreds of thousands of KIDMAP reports have been distributed to parents [28,29]. In this study, we implement KIDMAP to summarize patients' perception about each individual hospital's performances, distribute KIDMAP reports to hospital staff through the Internet, so that they can easily recognize key indicators for improvement. The reports also allow patients to compare hospitals' performance in quality of healthcare.

Quest [30] is the first software that produces KIDMAP reports. Chien et al. [31] developed a web-based KIDMAP (shortly Web-KIDMAP) on hospital indicator management, and they argued that Web-KIDMAP empowers diagnostic information by placing responses in four quadrants, not only for hospitals to improve their performances, but also for patients to better choose hospitals. 
Table I: Picker's 45-item inpatient questionnaire

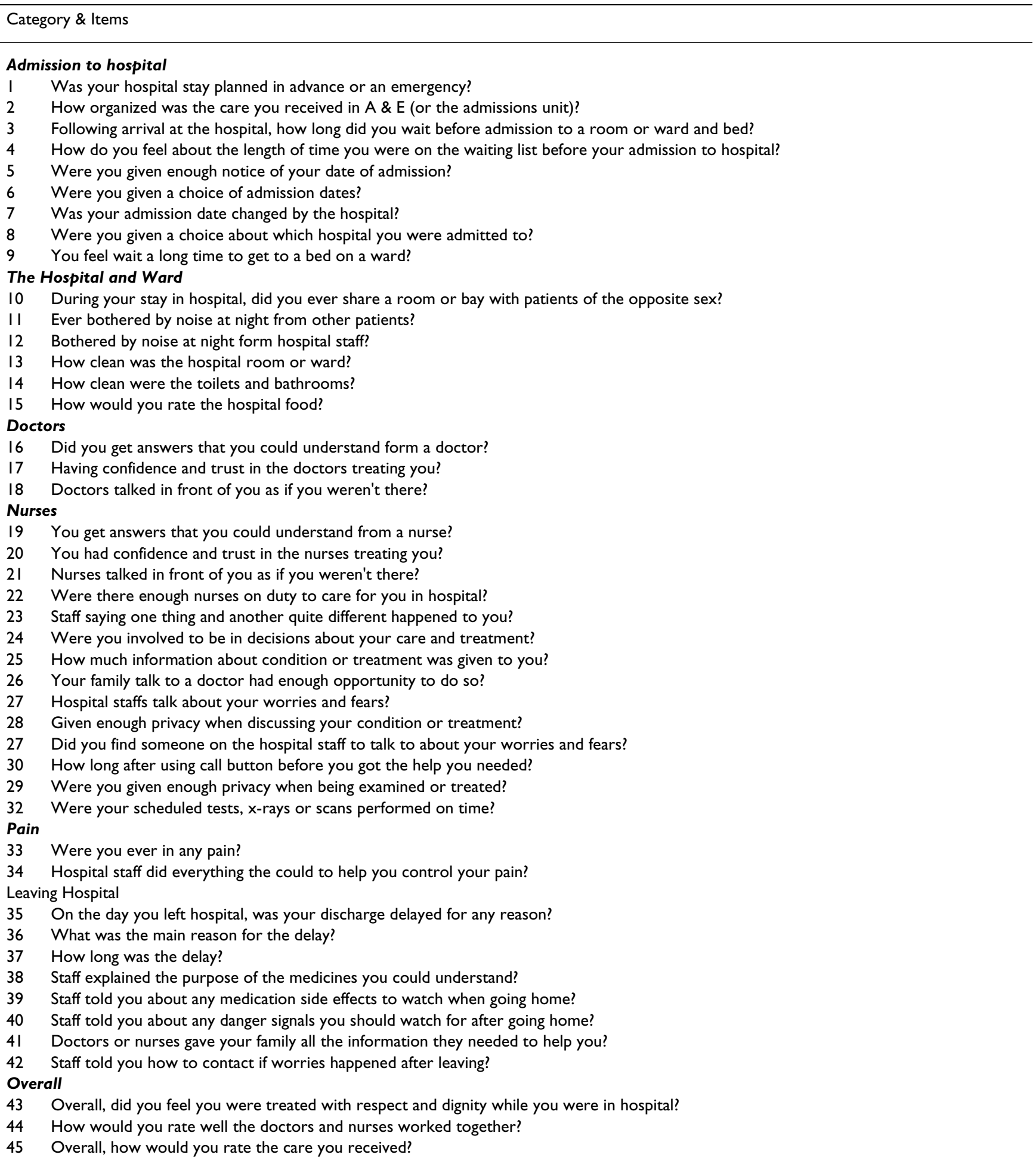

\section{(3) Aggregate scores produce useful results for questionnaires}

The EPIE questionnaires require patients to report what happened to them rather than how satisfied they are. EPIE believes that questionnaires about satisfaction do not pro- duce very useful results. A better approach is to require patients to report their experiences in hospitals so as to identify hospital strength and weakness, which allows hospitals to target problems and improve services [32]. Accordingly, an aggregate score for satisfaction level of 


\section{The hospital and ward}

Did you ever share a sleeping area with patients of the opposite sex?

Did you ever use the same bathroom or shower area as patients of the opposite sex?

Were you ever bothered by noise at night from other patients?

Were you ever bothered by noise at night from hospital staff?

In your opinion, how clean was the hospital room or ward that you were in?

How clean were the toilets and bathrooms that you used in hospital?

How would you rate the hospital food?

Were you offered a choice of food?

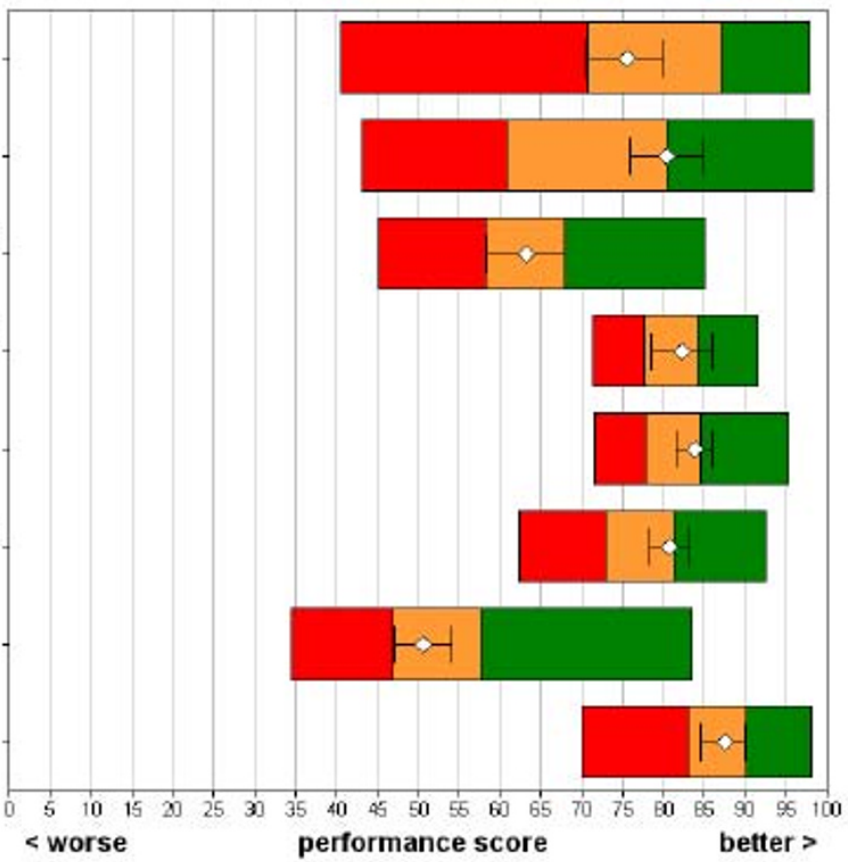

Doctors

When you had questions to ask a doctor, did you get answers you could understand?

Did you have confidence and trust in the doctors treating you?

Did doctors talk in front of you as if you weren't there?

As far as you know, did doctors wash or clean their hands between touching patients?

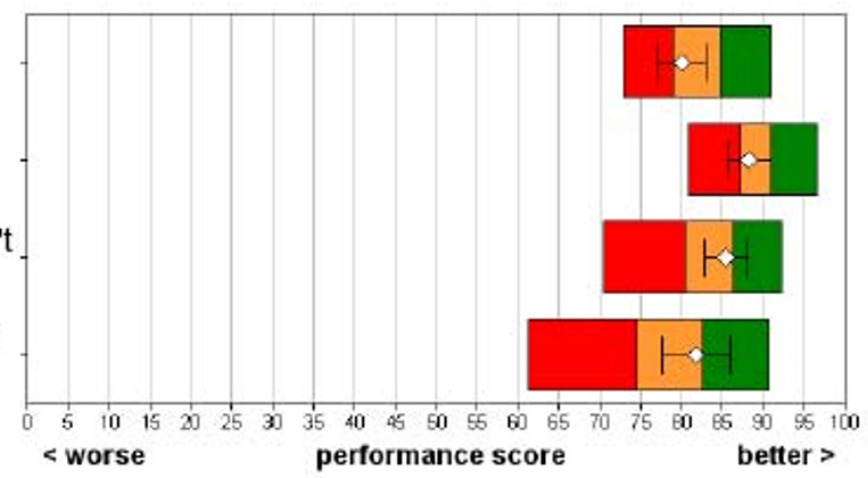

Best performing $20 \%$ of trusts Intermediate $60 \%$ of trusts

Worst performing $20 \%$ of trusts
This trust (vertical lines show amount of uncertainty as a result of random fluctuation)

This trust's results are not shown if there were fewer than 30 respondents.

Figure I

Item-by-item disclosures by the box plots. (Retrieved from http://www.webcitation.org/5WfaOHYOp). 
each hospital is essential and a quality control fit statistic indicator [33] is needed so that hospitals' performance can be quantified and compared.

\section{Aims of the study}

In this study, we apply Rasch analysis to (a) examine whether items in the EPIE questionnaire fit the model's expectations so that the underlying latent trait (henceforth referred to as satisfaction level) can be quantified and an interval scale can be obtained; (b) display the KIDMAP diagram $[28,29]$ on the web to help patients understand the overall performance of individual hospitals; and (c) quantify the performances among hospitals along a continuum single construct.

\section{Methods}

\section{Instrument and data collections}

With permission, we downloaded datasheets from a total of 169 hospitals in the 2003 English inpatient questionnaire from EPIE [17]. The original 45-indicator questions (Table 1) have been scored using a scale of 0 to 100 . The scores indicate the extent to which the patient's experience could have been improved. A score of 0 is assigned to all responses that reflect considerable need for improvement, whereas an answer option that has been assigned a score of 100 refers to a positive patient experience.

The questionnaire survey was carried out in all acute and specialist National Health Service (NHS) trusts (i.e., hospitals) in England that care for adult inpatients. Each of the 169 trusts, shown in Table 2, identified a list of 850 randomized eligible patients who had been discharged from the trust counting back from the last date of September, October or November 2003. The questionnaires were sent to 143,322 patients $(\cong 850 \times 169)$ and 88,308 completed questionnaires were returned. Patients were eligible to participate if they had had at least one overnight stay, were over 18 years old and were not maternity or psychiatry patients. Among the eligible patients, 54\% were women, $12 \%$ were aged 16 to 35 years, $17 \%$ were aged 36 to 50 years, $27 \%$ were aged 51 to 65 years, $33 \%$ were aged 66 to 80 years and $12 \%$ were aged 81 or over. The original data for the 88,308 patients were not available to us. Instead, the aggregated data for the 169 hospitals were shown on EPIE website for download and then analyzed by the authors.

\section{Data transformation and Rasch analysis}

\section{Data transformation}

We transformed the 45 items from a raw score of $0-100$ into five ordinal categories by a logarithm function of those original response data (i.e., integer $\log$ (original response raw score): 20, 60 and 100 to be 3, 4 and 5, respectively). From these five categories, the Rasch rating scale model [34] was fitted using the computer software
Table 2: Types of the 169 Hospitals in this study

\begin{tabular}{llr}
\hline Type & Type & Quantity \\
\hline Small & Small acute outside London & 27 \\
Small & Small acute London & 4 \\
Medium & Medium acute outside London & 38 \\
Medium & Medium acute London & 9 \\
Large & Large acute outside London & 39 \\
Large & Large acute London & 4 \\
Specialty & Acute specialist & 12 \\
Specialty & Orthopaedic & 4 \\
Teaching & Acute teaching outside London & 16 \\
Teaching & Acute teaching London & 9 \\
Large & Multi-service & 7 \\
\hline Total & & 169
\end{tabular}

WINSTEPS [35] with maximum likelihood estimation technique to yield a respective interval logit scaled score for individual hospitals [see Additional file 1], which is different from the traditional summed scores in ordinal nature as their satisfactory levels.

\section{Rasch analysis}

Rasch models [21] are latent trait models which imposes a probabilistic relationship between the level of latent trait (referred to as satisfaction level for a hospital in this study) and the items used for measurement (referred to as item difficulty). A fundamental assumption underlying Rasch models is unidimensionality, which can be assessed through point biserial correlation, Rasch fit indicators and Rasch factor analysis [36-38].

Rasch fit indicators include non-weighted (outfit) and weighted (infit) mean square errors (MNSQ) for items. The outfit MNSQ directly squares and averages standardized residuals; whereas the infit MNSQ averages standardized residuals with weights $[39,40]$. When items meet the model's expectations, their outfit or infit MNSQ will have an expected value of 1 . In contrast, an MNSQ very close to zero indicates redundancy of items and an MNSQ far lager than 1 indicates too much random noise.

For rating scales a range of 0.5 to 1.5 for the MNSQ statistics is often recommended as the critical range for a productive of measurement [41-43]. Items with an iutfit or infit MNSQ beyond this range are regarded as misfit. Successive Rasch analyses should be performed until all items satisfied the model fit requirements. Rasch model is superior to factor analysis in terms of confirming a factor structure [44]. When misfit items are identified and removed, unidimensionality is guaranteed and interval measures can be produced [45]. 
Principal components analyses (PCA) of the residuals from Rasch analysis can also be used to check the assumption of unidimensionality $[46,47]$. The following criteria are recommended to determine whether the assumption of unidimensionality holds: (a) the variance explained by the Rasch factor (the underlying construct) should be 4 times greater than that of the first principal component in the residuals; (b) the variance explained by the Rasch factor should be greater than $50 \%$; (c) the eigenvalues of the residuals should be smaller than 3; and (d) the percentage variance explained by the first principal component in the residuals should be less than $5 \%[41,48]$.

\section{Assessment of differential item functioning (DIF)}

To make comparison across different groups of hospitals, the test construct must remain invariant across groups. DIF analysis is a means to verify construct equivalence over groups [49]. If construct equivalence does not hold over groups, meaning that different groups respond to individual questions differently after holding their latent trait levels constant, then the estimated measures could not be compared directly over groups. In this study hospital type (small - large acute, teaching, etc) were examined for DIF. A $p$-value less than .05 indicates a DIF.

\section{KIDMAP shown on the web}

In order to improve the traditional KIDMAP profile, we developed an innovative web-based KIDMAP, called Web-KIDMAP, which reveals valuable information for hospital staff and patients in real time. Together with infit and outfit MNSQ, Web-KIDMAP reveals the strength and weakness of each hospital.

\section{Results}

\section{Examining a single underlying construct}

Among the 45 items, a total of 18 items (shown in Table 3 ) met the model's expectations fairly well (infit and outfit MNSQ within 0.5 and 1.5). These 18 items also covered those seven specified categories of Picker's original inpatient questionnaire. The 18 items had point-biserial correlations in a range from 0.66 to 0.84 . A principal component analysis on the residuals of Rasch scores showed no additional factors in that (a) the variance explained by items was more than 4 times greater than that of the first principal component (i.e. dividing $68 \%$ by $4.6 \%$ yields 14.78 times); (b) the variance explained by the Rasch factor was $68 \%$, greater than that the cutoff of $50 \%$ (c) the first eigenvalue was 2.6, less than the cutoff of 3 ; and (d) the percentage of variance explained by the first principal component was of $4.6 \%$, less than $5 \%$. These results indicated that there was a good model-data fit and that the assumption of unidimensionality held for these 18 polytomous items. The category Rasch-Andrich thresholds (step difficulties) were ordered as $-3.76,-1.91,1.57$ and 4.11 .
Overall, these 18 items exhibited a good model-data fit. Hence, they measured a single construct for patient satisfaction and an interval scale of logits was achieved for further comparison and analysis [38]. The hospital measures ranged from -1.59 to 9.63 with mean 2.64 and standard deviation 2.09, indicating items were easier for these hospitals and a wide range of hospitals dispersed on the interval scale.

The hospital sample separation reliability was 0.94 (Cronbach's $\alpha[50]=0.96)$, indicating that these 18 items could differentiate the hospitals very well. The separation index for the items (a measure of the spread of the estimates relative to their precision) was as high as 4.01 , allowing us to differentiate between five statistically distinct strata of item difficulties with the formula of strata $=(4 \times 4.01+$ 1)/3 [51].

Analysis of variance on the hospital measures reveals a significant difference $(F=8.318 ; p<.001)$ among types of hospitals: General practices $(M=4.72$ logits $)$ performed the best, followed by Large hospitals ( $M=3.47$ logits), Teaching hospitals $(M=2.62$ logits $)$, Small hospitals $(M=$ 2.25 logits), and Medium hospitals ( $M=.82$ logits $)$.

\section{Item properties}

The three most difficult items to be satisfied by patients were: item 39 (Staff told you about any medication side effects to watch when going home), item 41 (Doctors or nurses gave your family information needed to help you) and item 27 (Hospital staff talks about your worries and fears). The easiest one was item 34 (Hospital staff did everything they could to help you control your pain). The mean and standard deviation of items was 0.00 and 1.52, respectively. All of items were in a range of absolute 4.9 logits. The item difficulties were well spread out across the hospitals, indicating that these items could differentiate hospitals fairly well so as to reach a hospital separation reliability of 0.94 .

Item invariance refers to the fact that the estimated item location parameters should not depend on the sample used to calibrate the estimates [50]. Table 3 shows that no DIF items were found across different types of hospitals, suggesting these items measure the same construct across types of hospitals such that their performances can be directly compared.

\section{KIDMAP used for diagnosing hospitals}

Figure 2 shows a Web-KIDMAP for a particular hospital. In the right-hand bottom corner (the 4th quadrant), there are six 'easier not achieved' items that the hospital was expected to have achieved given the performance estimate of 6.14 logits and the percentile rank of 10 (see to the right of the percentile column in Figure 2). 
Table 3: Item difficulties with DIF-free and Fit MNSQ statistics of the 15 items in the 2003 English inpatient questionnaire of the Picker Institute Europe

\begin{tabular}{|c|c|c|c|c|c|c|c|}
\hline \multirow[t]{2}{*}{ No. } & \multirow[t]{2}{*}{ Category \& Item } & \multicolumn{2}{|c|}{ Difficulty } & \multicolumn{2}{|c|}{ MNSQ } & \multirow[b]{2}{*}{ PTME§ } & \multirow{2}{*}{$\begin{array}{l}\text { DIF-free } \\
\text { P-value* }\end{array}$} \\
\hline & & Logit & $S E$ & Infit & Outfit & & \\
\hline & Admission to hospital & & & & & & \\
\hline 2 & How organized was the care you received in A\&E? & -0.95 & 0.15 & 1.05 & 1.18 & 0.7 & .736 \\
\hline 5 & Were you given enough notice of your date of admission? & -1.08 & 0.15 & 1.02 & 0.94 & 0.69 & .968 \\
\hline \multirow[t]{2}{*}{9} & You feel wait a long time to get to a bed on a ward? & -0.63 & 0.14 & 1.2 & 1.1 & 0.74 & .116 \\
\hline & The Hospital and Ward & & & & & & \\
\hline 11 & Ever bothered by noise at night from other patients? & 1.58 & 0.13 & 1.50 & 1.50 & 0.74 & .289 \\
\hline \multirow[t]{2}{*}{12} & Bothered by noise at night form hospital staff? & -1.1 & 0.15 & 1.02 & 1.02 & 0.69 & .287 \\
\hline & Doctors & & & & & & \\
\hline 17 & Having confidence and trust in the doctors treating you? & -1.1 & 0.15 & 0.61 & 0.66 & 0.79 & .553 \\
\hline \multirow[t]{2}{*}{18} & Doctors talked in front of you as if you weren't there? & -1.12 & 0.15 & 1.12 & 1.07 & 0.67 & .120 \\
\hline & Nurses & & & & & & \\
\hline 19 & You get answers that you could understand from a nurse? & -1.12 & 0.15 & 0.56 & 0.57 & 0.8 & .114 \\
\hline 23 & Staff saying one thing and another quite different happened to you? & -1.1 & 0.15 & 0.98 & 0.89 & 0.71 & .199 \\
\hline 24 & Were you involved to be in decisions about your care and treatment? & 0.67 & 0.14 & 0.77 & 0.77 & 0.84 & .068 \\
\hline 27 & Hospital staff talk about your worries and fears? & 2.22 & 0.13 & 0.56 & 0.52 & 0.81 & .364 \\
\hline \multirow[t]{2}{*}{30} & How long after using call button before you got the help you needed? & 0.42 & 0.14 & 0.91 & 1.21 & 0.66 & .253 \\
\hline & Pain & & & & & & \\
\hline \multirow[t]{2}{*}{34} & Hospital staff did everything they could to help you control your pain? & -1.12 & 0.15 & 0.82 & 0.87 & 0.74 & .906 \\
\hline & Leaving Hospital & & & & & & \\
\hline 38 & Staff explained the purpose of the medicines you could understand? & -1.1 & 0.15 & 0.97 & 0.87 & 0.71 & .972 \\
\hline 39 & Staff told you about medication side effects when going home? & 3.78 & 0.13 & 1.22 & 1.16 & 0.81 & .407 \\
\hline 41 & Doctors or nurses gave your family information needed to help you? & 2.76 & 0.13 & 1.37 & 1.44 & 0.78 & .090 \\
\hline \multirow[t]{2}{*}{42} & Staff told you how to contact if worries happened after leaving? & -0.3 & 0.14 & 1.33 & 1.19 & 0.73 & .344 \\
\hline & Overall & & & & & & \\
\hline \multirow[t]{3}{*}{44} & How would you rate well the doctors and nurses worked together? & -0.71 & 0.14 & 0.75 & 0.67 & 0.8 & .303 \\
\hline & Mean & 0.00 & 0.14 & 0.99 & 0.98 & 0.75 & \\
\hline & $S D$ & 1.56 & 0.01 & 0.28 & 0.28 & 0.05 & \\
\hline
\end{tabular}

Note. *DIF CHI-square probability from TABLE 30.4 of WINSTEPS software

§biserial correlations

The most unexpected errors among the three items, one noted with an asterisk $\left({ }^{*}\right)$ and two with a caret $\left({ }^{\wedge}\right)$, are indicated as statistically significantly different $(p<0.05$ and $p<0.01$, respectively). The label 6.5 .4 in the 4 th quadrant means that the $5^{\text {th }}$ category of item 6 was endorsed as 4 by the hospital. Actually, the hospital (6.41 logits) had a very good chance to achieve a score higher than 4 but failed to achieve (e.g., see the left-hand side in Figure 2). These unexpected responses are informative and worth noting, because the hospital's weakness did not match the patients' perception. Note that the unexpected response was identified by inspecting the hospital's own performance level (i.e., self comparison), rather than the averaged scores in Picker's item-by-item diagram, Figure 1 .

\section{Three steps to read the Web-KIDMAP}

We analyzed the data from the 2003 EPIE inpatient questionnaire [17] and developed a Web-KIDMAP diagram that could be visualized on the Internet for (a) inter-hospital comparison (by inspecting performance levels along the logit scale), (b) intra-hospital comparison (by inspect- ing response patterns and residual Z-scores), and (c) model-data fit checking (by inspecting MNSQ statistics).

\section{Discussion \\ Findings}

We use Rasch measurement to construct an interval logic scale for patient satisfaction on hospital performance, utilize fit statistics to detect aberrant response pattern, and develop a visual representation on website, Web-KIDMAP, to overcome the drawback in traditional individual item-by-item box plots of disclosure for patient views on hospital service quality.

The item-by-item analysis of the Picker's questionnaire ignores measurement error that is embedded in an item. In reality, a self-report item (like those in Picker's questionnaire) is very imprecise and contains a large measurement error, which invalidates item-by-item analysis. Besides measurement error, item-by-item analysis is useful only when each provides feedback information about an individual practice (or program, treatment, etc.). It is hard to believe that there are 45 kinds of practice that have 


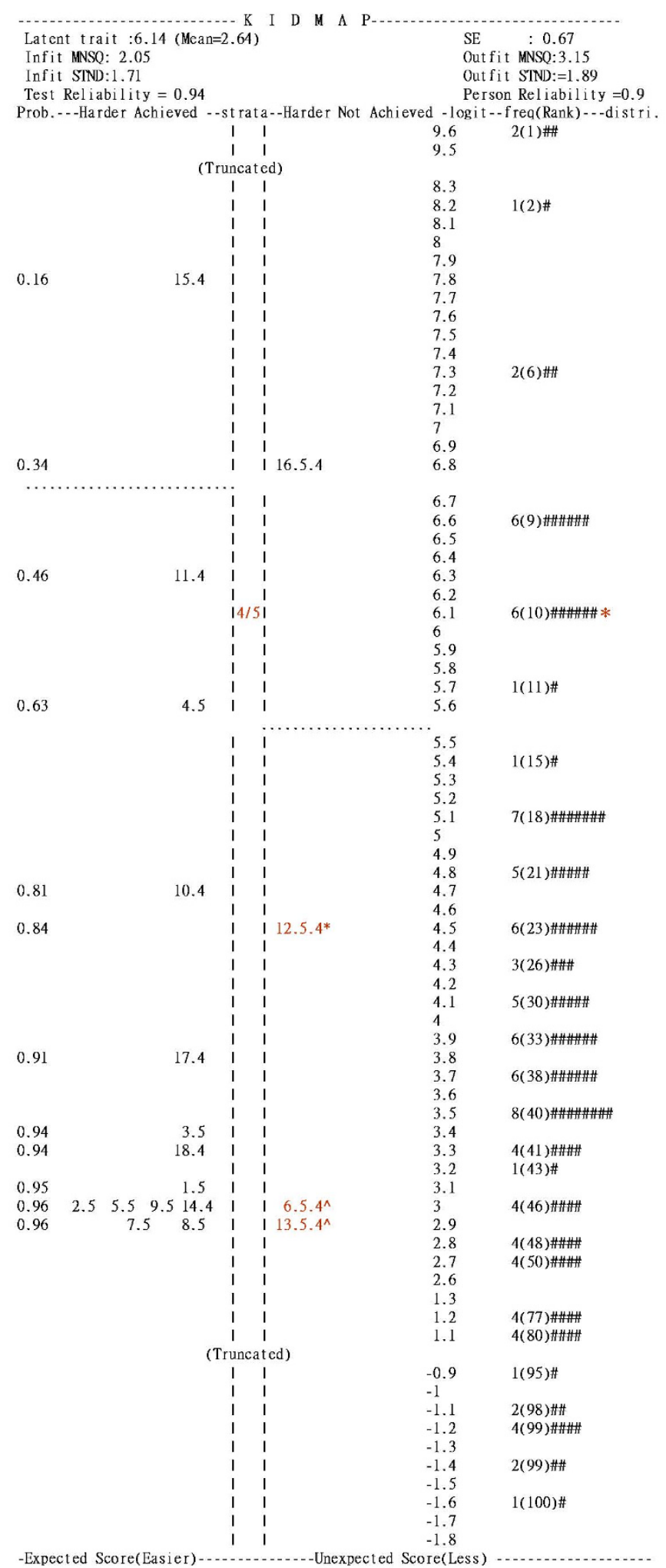

Figure 2

KIDMAP profile of an actual assessed hospital. 
been routinely carried out in a hospital, such that we need 45 items to provide feedback information, one item for one kind of practice.

Rasch analysis considers measurement error in an item and yields global and local feedback information for hospital performance (e.g., item and person fit analysis, DIF analysis, item difficulty hierarchy, the Wright map, the KIDMAP, etc.).

\section{Strengths of the study}

I. Objective measurement by Item-fitting with the Rasch model

Using the Rasch approach, we have examined patient experience from the EPIE 2003 inpatient questionnaire. Of the original 45 items, 18 items meet the model's expectations fairly well so that the underlying satisfaction latent trait is successfully quantified via the 18 items. It turns out that the most difficult area for hospitals to earn inpatients' satisfaction is "Staff told you about any medication side effects to watch when going home."

\section{KIDMAP based on interval logit scores yielded by Rasch analysis} KIDMAP is derived from performance diagnosis of students in education fields $[28,29]$. It is based on an interval scale constructed by Rasch analysis. The Web-KIDMAP approach is invented by the authors as a means to perform macro- and micro-examinations of hospital performance [31], to provide much more valuable information than that generated by the traditional Quest software [30]. In this study we show how to construct a visualized program of Web-KIDMAP in order to describe a hospital's overall performance, diagnose its response patterns, and then transplant all the information onto a website.

\section{Quality control Item fit statistics}

The strength (on the top-left corner in Figure 2) and weakness (on the bottom-right corner in Figure 2) of the hospitals are displayed on Web-KIDMAP to allow hospital staff to generate performance improvement plans and patients to select hospitals. Web-KIDMAP engenders a basis of intra- and inter-hospital comparison in quality of healthcare: considering why the weakness responses are endorsed by patients, what is warranted is further support the strengthened indicators, and what possible tools or methods are called for to satisfy patients' targeted expectations in the top-right corner (the 1st quadrant of Figure 2) on which the performance ability estimate could be upgraded to a level higher than 6.14 logits in Figure 2.

\section{Limitations of the study}

High quality healthcare feedback is important. More work is needed to further enable the administration of an effective feedback system in healthcare settings. In this study, we demonstrate how a visualized Web-KIDMAP can be used to compare patient perception on healthcare service on the Internet. Users may need some background knowledge to interpret Web-KIDMAP properly.

Due to DIF and misfit to the model, 27 items are removed. This is because these items do not work with the other items harmoniously to measure the same construct, not because they are useless or unimportant to hospital performance. Future studies can investigate importance of these 27 items. If they are very important to hospital performance, then a stand-along scale should be developed. Future study can further macro-examine the test properties and to micro-investigate the item response with/without errors or abnormalities [52].

\section{Applications}

KIDMAP is not designed to replace traditional item-byitem box plots as shown in Figure 1, but to complement them. Furthermore, Web-KIDMAP is highly dynamic and easily interpretable with graphic tools as well as the exemplary comparisons in quality of healthcare, especially on the Internet referring to Figure 3.

Fit MNSQ statistics describe measurement quality. As for the illustration in Figure 2, an outfit MNSQ of 3.15 indicates that the patients' responses to the hospital does not provide useful information and there is too much unexplained noise in the observation (such as those three red colored items in Figure 2)[52].

The indicators in the top-left corner (the 2nd quadrant) are the hospital's strength associated with the category 'harder ones but achieved'. The probability of endorsing such an item by the hospital is shown in the ultimate left column. " $4 / 5$ ", located in the middle of the Web-KID$\mathrm{MAP}$, indicating the performance estimate on location of 6.14 logits classified as the 4th best stratum (note: the separation index for the persons is as high as 4.01 , allowing us to differentiate between five statistically distinct strata of person abilities with the formula of strata $(=(4 \times 4.01$ $+1) / 3$ ). Percentile ranks, frequencies and the distribution of the norm-reference from 169 hospitals are innovated and shown on the right-hand side, differing from the traditional KIDMAP provided by the Quest software [30].

\section{Further studies and suggestions}

Web-KIDMAP provides a response profile of an individual hospital. Hospital staff can use Web-KIDMAP to inspect patients' perception about hospital performance. Aberrant responses in those items of "easier not achieved" deserve more attention. The Z-scores in Figure 2 provide an opportunity for a hospital to examine its strength and weakness by self-comparison rather than compared with other hospitals, so as to upgrade the overall performance level. 


\section{England inpatient questionnaire of the Picker Institute Europe}

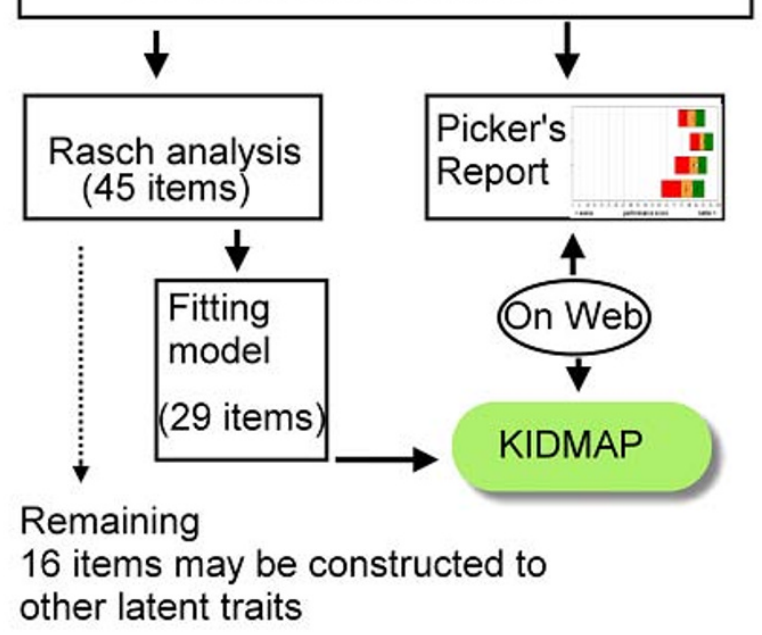

Figure 3

Flow chart of this study.

The transformation of ordinal raw scores into interval logits using Rasch measurement [21.38] within patient-centered research is worth further research. We believe that Web-KIDMAP [31] is indicative, efficient and effective and can easily facilitate patient-centered clinical environment.

We herein propose that healthcare informatics be regarded as a whole newly integrated academic discipline, one that should be devoted to the exploration of the new possibilities that informatics is creating for both hospital staff and patients in relation to health and healthcare issues $[53,54]$.

These 18 items can be added to a static questionnaire as a daily routine for examining healthcare quality of a hospital or as item banks created for a touch screen version of computer adaptive testing (CAT) to reducing patient burdens in responding questions [55], which studies are worth carrying out in the future.

\section{Conclusion}

The England Picker Institute Europe annually discloses reports of patients' experience with a particular provider at a specific point by an item-by-item approch with box plots indiividually. In this study, we apply IRT-based Rasch analysis to create Web-KIDMAP to help patients understand the overall performance of individual hospitals, and quantify the performances among hospitals along an interval scale. Web-KIDMAP provides an newly developed comparison in quality of healthcare and allows intra- and inter-hospital comparison on the Internet.

\section{Abbreviations}

IRT: item response theory; MNSQ: mean square error; NHS: National Health Services.

\section{Competing interests}

The authors declare that they have no competing interests.

\section{Authors' contributions}

TW designed the study and performed the analysis, interpreted the results, and drafted the manuscript. WC contributed to the revision of manuscript. HY helped to interpret the results and participated in the formulation of the discussion. HJ helped to interpret the results and contributed to the discussion. All authors reviewed and edited the manuscript for intellectual.

\section{Additional material}

\section{Additional file 1}

The control file of WINSTEPS software. The data provided represent the Rasch analysis of the 18 items fitting to the model's expectations.

Click here for file

[http://www.biomedcentral.com/content/supplementary/14726963-9-135-S1.txt]

\section{Acknowledgements}

This study was supported by Grant CMFHR9820 from Chi Mei Medical Center, Taiwan.

\section{References}

I. Chen J, Radford MJ, Wang Y, Marciniak TA, Krumholz HM: Do America's Best Hospitals perform better for acute myocardial infarction?". N Engl J Med I999, 340(4):286-92.

2. Eysenbach G, Thomson M: The FA4CT algorithm: a new model and tool for consumers to assess and filter health information on the Internet. Medinfo 2007, I 2(Pt I): I42-6.

3. Eysenbach G: From intermediation to disintermediation and apomediation: new models for consumers to access and assess the credibility of health information in the age of Web2.0. Medinfo 2007, I 2(Pt I): 162-6.

4. Eysenbach G: Medicine 2.0. Social Networking, Collaboration, Participation, Apomediation, and Openness. J Med Internet Res 2008, 10(3):e22.

5. Krumholz HM, Rathore SS, Chen J, Wang Y, Radford MJ: Evaluation of a consumer-oriented internet health care report card: the risk of quality ratings based on mortality data. JAMA 2002, 287(10): 1277-87.

6. Judith KB, Tierney EG, Shoshanna S, Cathy ED, William JW, Marcia KP: Using Public Reports of Patient Satisfaction for Hospital Quality Improvement. Health Serv Res 2002, 4 I (3 Pt I):663-682.

7. Tuffs A: German hospital rankings questioned. British Medical Journal 2007, 335:177.

8. Wang OJ, Wang Y, Lichtman JH, Bradley EH, Normand SL, Krumholz HM: America's Best Hospitals" in the treatment of acute myocardial infarction. Arch Intern Med 2007, I67(13): 1345-5I.

9. O'Brein SM, Peterson ED: Identifying high-quality hospitals: consult the ratings or flip a coin?". Arch Intern Med 2007, 167:1342-4. 
10. Picker (Picker Institute Europe): What do you think of your doctor? [http://www.pickereurope.org/]. accessed September 22, 2008

II. Leapfrog Group [http://www.leapfroggroup.org/]. accessed September 22, 2008

12. Patient Opinion [http://www.patientopinion.org.uk/]. accessed September 22, 2008. accessed September 22, 2008

13. Barreto C: What comes after Web 2.0? [http://www.technologyre view.com/Infotech/17845/]. accessed September 22, 2008

14. Giustini D: How web 2.0 is changing medicine. British Medical Journal 2006, 333: I 283-4.

15. Lilford RJ, Brown CA, Nicholl J: Use of process measures to monitor the quality of clinical practice. British Medical Journal 2007, 335:648-50.

16. Magee H, Davis LJ, Coulter A: Public views on healthcare performance indicators and patient choice. J R Soc Med 2003 96(7):338-380.

17. EPIE: Patient survey report - adult inpatients Picker Institute Europe, Oxford; 2004

18. Coulter A: The evidence speaks for itselfbut can the public? Nursing Management 2003, I I(8): I 2-I5.

19. Likert RA: Technique for the Measurement of Attitudes. Archives of Psychology 1932, 140:32-66.

20. Johnson DE: Applied multivariate methods for data analysis Duxbury, Belmont, CA; 1998.

21. Rasch G: Probabilistic Models for Some Intelligent and Attainment Tests Copenhagen, Denmark: Institute of Educational Research; 1960.

22. Schuster MA, McGlynn EA, Brook RH: How good is the quality of health care in the United States? Milbank $Q$ 1998, 76:5 I7-580.

23. Seddon ME, Marshall MN, Cambell SM, Roland MO: Systematic review of quality if clinical care in general practice in the UK, Australia and New Zealand.". Qual Health Care 2001, 10:152-160.

24. Stevens SS: On the theory of scales of measurement. Science 1946, I 03:677-680.

25. Doran T, Fullwood C, Gravelle H, Reeves D, Kontopantelis E, Hiroeh $U$, Roland M: Pay-for-performance programs in family practices in the United Kingdom. The New England Journal of Medicine 2006, 355(4):375-384.

26. Stout WF: A new item response theory modeling approach with applications to unidimensionality assessment and ability estimation. Psychometrika 1990, 55(2):293-325.

27. Lord FM, Novick MR: Statistical theories of mental test scores. Reading, MA: Addison-Wesley; 1968.

28. Wright BD, Mead RJ, Ludlow LH: KIDMAP: Person-by-item interaction mapping. In Research Memorandum MESA Psychometric Laboratory, Department of Education, University of Chicago, Chicago; 1980:29.

29. Masters GN: KIDMAP - a history. Rasch Measurement Transaction 1004, 8(2366 [http://www.rasch.org/rmt/rmt82k.htm]. accessed September 22, 2008. accessed 2008 Jan. 25

30. Adams RJ, Khoo ST: QUEST - Version 2.I The Interactive Test Analysis System. Rasch Measurement Transactions 1998, I I (4):598.

31. Chien TW, Wang WC, Chen NS, Lin HJ: Improving Hospital Indicator Management with the Web-KIDMAP Module: THIS as an Example. Journal of Taiwan Association for Medical Informatic 2006 I5(4): 15-26.

32. Jenkinson $C$, Coulter A, Bruster S, Richards N, Chandola T: Patients' experiences and satisfaction with health care: results of a questionnaire study of specific aspects of care. Qual Saf Health Care 2002, I I (4):335-344.

33. Smith RM: A comparison of methods for determining dimensionality in Rasch Measurement. Structural Equation Modeling 1996, 3(1):25-40.

34. Andrich DA: rating scale formulation for ordered response categories. Psychometrika 1978, 43:56I-73.

35. Linacre JM: WINSTEPS [computer program], Chicago, IL [http:// www.WINSTEPS.com]. accessed September 22, 2008

36. Linacre JM: Detecting multidimensionality: which residual data-type works best? Journal of Outcome Measurement 1998 2(3):266-283.

37. Bond TG, Fox CM: Applying the Rasch model: Fundamental measurement in the human sciences. 2 nd edition. Mahwah, NJ: Erlbaum; 2007.

38. Linacre JM: Structure in Rasch residuals: Why principal components analysis? Rasch Measurement Transactions 1998, I 2(2):636.
39. Wright BD, Masters GN: Rating Scale Analysis. Chicago, III: MESA Press; 1982.

40. Wright BD, Mok M: Understanding Rasch Measurement: Rasch Models Overview. J Appl Meas 2000, I:83-106.

4I. Linacre JM: User's guide to WINSTEPS. Chicago: Mesa Press; 2009.

42. Schumacker RE, Linacre JM: Factor analysis and Rasch analysis. Rasch Measurement Transactions 1996, 9(4):470.

43. Wright BD, Linacre JM, Gustafson J-E, Martin-Lof P: Reasonable mean-square fit values. Rasch Meas Trans 1994, 8:370.

44. Waugh RF, Chapman ES: An analysis of dimensionality using factor analysis (true-score theory) and Rasch measurement: What is the difference? Which method is better? J Appl Meas 2005, 6:80-99.

45. Smith EV Jr: Detecting and evaluating the impact of multidimensionality using item fit statistics and principal component analysis of residuals. J Appl Meas 2002, 3(2):205-3I.

46. Smith AB, Wright EP, Rush R, Stark DP, Velikova G, Selby PJ: Rasch analysis of the dimensional structure of the Hospital Anxiety and Depression Scale. Psychooncology 2006, I 5(9):8|7-27.

47. Smith $A B$, Wright $P$, Selby PJ, Velikova G: A Rasch and factor analysis of the Functional Assessment of Cancer Therapy-General (FACT-G). Health Qual Life Outcomes 2007, 5: I9.

48. Wright BD, Stone MH: Making measures. Chicago, The Phaneron Press; 2004.

49. Holland PW, Wainer H: Differential Item Functioning. Hillsdale NJ: Erlbaum; 1993.

50. Cronbach LJ: Coefficient alpha and the internal structure of tests. Psychometrika 1951, 16:297-334.

51. Fisher WP: Reliability and separation. Rasch Measurement Transactions 1996, 9:472

52. Linacre JM: Optimizing rating scale category effectiveness. Appl Meas 2002, 3(I):85-106

53. Eysenbach G, Jadad AR: Evidence-based patient choice and consumer health informatics in the Internet age. J Med Internet Res 2001, 3(2):el9.

54. Blais MA: Have you heard we're having a revolution? The coming of modern test theory. Journal of Personality Assessment 2003, 80(2):208-210.

55. Chien TW, Wu HM, Wang WC, Castillo RV, Chou W: Reduction in patient burdens with graphical computerized adaptive testing on the ADL scale: tool development and simulation. Health Qual Life Outcomes 2009, 7:39.

\section{Pre-publication history}

The pre-publication history for this paper can be accessed here:

http://www.biomedcentral.com/1472-6963/9/135/pre $\mathrm{pub}$
Publish with Bio Med Central and every scientist can read your work free of charge

"BioMed Central will be the most significant development for disseminating the results of biomedical research in our lifetime. "

Sir Paul Nurse, Cancer Research UK

Your research papers will be:

- available free of charge to the entire biomedical community

- peer reviewed and published immediately upon acceptance

- cited in PubMed and archived on PubMed Central

- yours - you keep the copyright
BioMedcentral 